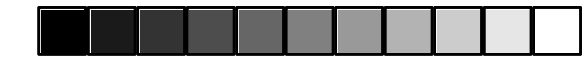

\title{
LOS BANCOS CENTRALES COMO PRESTAMISTAS DE ULTIMA INSTANCIA*
}

\author{
Fernando Ossa S.**
}

\begin{abstract}
Central banks act as lenders of last resort to commercial banks and other financial institutions. However, there is much controversy about whether or not they should perform this function. This article analyses, in the first place, the principal aspects of this controversy. Furthermore, the classical version of the lender of last resort is presented, according to the recommendations of Thorton and Bagehot, together with an analysis of the way in which central banks have acted in practice as lenders of last resort. Finally there is a brief discussion on how this function could be extended to the international context.
\end{abstract}

\section{RESUMEN}

Los bancos centrales actúan como prestamistas de última instancia de los bancos comerciales y otras instituciones financieras. Sin embargo, existe bastante controversia sobre si es conveniente o no que ejerzan esta función. En este artículo se analizan, en primer lugar, los aspectos principales de esta controversia. Además, se presenta la versión clásica del prestamista de última instancia, representada por las recomendaciones de Thorton y Bagehot, y se analiza cómo han actuado en la práctica los bancos centrales en su función de prestamistas de última instancia. Finalmente se discute brevemente cómo esta función se puede extender al contexto internacional.

* Agradezco los comentarios de Gert Wagner.

** Profesor Instituto de Economía, Pontificia Universidad Católica de Chile. Email:fossa@faceapuc.cl

Keywords: Lender of Last Resort, Central Banks, Commercial Banks, Financial Crises, International Monetary Fund.

JEL Classification: E58, F33, G15, G21 


\section{INTRODUCCIÓN}

En el siglo XIX se publicaron los trabajos clásicos de Thorton (1802) y Bagehot (1873), quienes argumentaron que el Banco de Inglaterra debía apoyar a los bancos comerciales en problemas, actuando como prestamista de última instancia para evitar el contagio entre bancos y los pánicos bancarios.

Aunque los bancos centrales en general actúan como prestamistas de última instancia, existe bastante controversia sobre si es conveniente o no que ejerzan esta función. Los objetivos de este artículo son presentar los aspectos principales de esta controversia, referirse a la visión clásica del prestamista de última instancia representada por las recomendaciones de Thorton y Bagehot, y analizar cómo han actuado en la práctica los bancos centrales en su función de prestamistas de última instancia.

Comenzamos, en la segunda sección, por referirnos al origen de los bancos centrales y al paso de los sistemas de patrón metálico al patrón fiduciario que rige en el presente. También se discuten en esta sección las características de las cámaras de compensación, que en algunos países precedieron a los bancos centrales, y que ejercían algunas de las funciones que éstos después asumieron.

En la tercera sección se presentan los aspectos principales de las dos posiciones en la controversia sobre si se justifica o no la función de prestamista de última instancia. En esta parte se hace referencia también a otros mecanismos que buscan estabilizar al sistema bancario, como los seguros de depósitos y la separación de las funciones de los bancos en dos organizaciones distintas.

En la cuarta parte se presenta la visión clásica del prestamista de última instancia, según los principios enunciados por Thorton y Bagehot en el siglo XIX.

La quinta sección estudia la forma cómo han actuado los bancos centrales en la práctica, en su función de prestamistas de última instancia, y cómo se relaciona esto con la visión clásica. Aquí se hace referencia también a la política de "ambigüedad constructiva" adoptada por algunos bancos centrales respecto a su posible ayuda a los bancos comerciales.

Aunque este artículo se centra básicamente en la función de prestamista de última instancia a nivel nacional, en la sección quinta y final se discute brevemente cómo esta función puede extenderse al ámbito internacional. Esto introduce el tema de las crisis financieras internacionales, la nueva arquitectura financiera internacional y la posibilidad de que el Fondo Monetario Internacional actúe como prestamista de última instancia a nivel internacional. 


\section{El origen de los Bancos Centrales}

Los primeros bancos centrales se originaron en Europa ${ }^{1}$. En un comienzo no se dio mayor importancia a su posible función de supervisión y apoyo a los otros bancos, debido a que los gobiernos estaban más bien interesados en los recursos financieros que podían obtener de los bancos centrales.

Algunas de estas instituciones eran dominadas por el Estado, como sucedía con el Banco de Prusia y del Banco de Francia. En otros casos se trataba de bancos privados, como el Banco de Inglaterra, que sólo fue nacionalizado después de la segunda guerra mundial. A cambio del apoyo financiero al gobierno, los Banco Centrales recibían ciertos privilegios, como el monopolio de la emisión de billetes convertibles en oro o plata. Su posición dominante se tradujo en una centralización de las reservas de oro o plata en estas instituciones, las que empezaron a actuar como banco de los otros bancos. En algunos casos se generaron conflictos, cuando actuaban como un competidor de los otros bancos. Pero esto fue cambiando a lo largo del tiempo, a medida que el objetivo de obtención de utilidades fue perdiendo importancia, y los Banco Centrales pusieron el énfasis en la mantención de la convertibilidad y en la estabilidad de los otros bancos, para los cuales actuaban como prestamistas de última instancia.

La mayoría de los bancos centrales que se establecieron fuera de Europa, principalmente a fines del siglo XIX y en las primeras décadas del siglo XX, fueron desde un comienzo instituciones no competitivas con los otros bancos y sin un objetivo de maximización de utilidades.

La función de prestamista de última instancia adoptada por los bancos centrales significó un seguro implícito para los bancos comerciales. En consecuencia se introdujo la posibilidad de riesgo moral, en la medida que los bancos y sus depositantes incurriesen en riesgos excesivos, esperando ser rescatados por el Banco Central en caso de necesidad. Esto, a su vez, hizo que los bancos centrales se involucrasen, directa o indirectamente, en la regulación y supervisión de los bancos comerciales.

En ciertos casos, como en Estados Unidos, antes de crearse el Banco Central, algunas de sus funciones eran ejercidas por las cámaras de compensación. Estas se crearon inicialmente para compensar los cheques de los bancos miembros. Pero las cámaras actuaron también como prestamistas de última instancia y ejercieron control sobre los bancos participantes, a través de tasas de encaje mínimas, requisitos de capital, auditorías, etc. ${ }^{2}$ Si un banco no cumplía con las reglas, recibía sanciones que iban desde multas hasta la expulsión de la cámara. De esta forma se enfrentaban los problemas de exceso de riesgo por parte de los

1 Sobre la evolución de los principales bancos centrales, véanse Goodhart (1988), Schuler (1996) y Fry, Goodhart y Almeyda (1996). Algunos bancos centrales se originaron en bancos comerciales que fueron adoptando funciones de banco central a lo largo de un proceso evolutivo, mientras que otros fueron creados desde un comienzo como bancos centrales con privilegio de emisión de billetes.

2 Véanse Gordon y Mullineaux (1987), David (1994) y Ossa (2002). 
bancos. En el caso de pánico generalizado la cámara emitía “certificados de préstamos" que los bancos recibían a cambio de activos y que podían usar en el proceso de compensación, lo cual liberaba oro que quedaba disponible para hacer frente a los retiros del público. Incluso, en algunas ocasiones en que no alcanzaba el oro para hacer frente a los retiros, se entregaron "certificados de préstamo" al público, el cual los aceptaba ya que no eran derechos sobre un banco específico, sino que sobre la cámara en su conjunto. Otro mecanismo usado por las cámaras en situaciones de pánico generalizado fue la suspensión temporal de la convertibilidad de los depósitos en oro. Esto resultaba beneficioso para los bancos y para los depositantes cuando estos últimos tenían información incompleta sobre los bancos. La cámara, que tenía mejor información, usaba la suspensión de la convertibilidad para dar una señal a los depositantes de que era en el beneficio de ambos que no se retiraran los depósitos.

Pero las cámaras de compensación también pueden ser consideradas como organizaciones monopólicas que cobran comisiones excesivas por su labor de intermediación. Esto último puede haber sido una de las motivaciones para la estatización de las funciones de las cámaras de compensación y la creación de un Banco Central en algunos países.

Los principales bancos centrales operaron bajo un sistema de convertibilidad metálica hasta la primera guerra mundial. El patrón oro se restableció en la década de 1920, pero fue, en general, abandonado en la década de 1930 como consecuencia de la crisis de 1929. Después de la segunda guerra mundial se mantuvo la inconvertibilidad de los billetes en oro. Pero los países adoptaron el sistema de tipo de cambio fijo respecto al dólar norteamericano, el cual era externamente convertible en oro, es decir, para instituciones oficiales no residentes. Con el abandono de la convertibilidad externa del dólar en oro y el término del sistema de paridades fijas a comienzo de los años 70, en la actualidad los países se encuentran bajo un patrón fiduciario con libertad para escoger su sistema cambiario. Esto significa que existen menos limitaciones para que los bancos centrales actúen como prestamistas de última instancia.

\section{Controversias sobre la Existencia de un Prestamista de Ultima INSTANCIA}

Existe una controversia sobre si se justifica que los bancos centrales actúen como prestamistas de última instancia o si, alternativamente, hay otras soluciones para los problemas que pueden enfrentar los bancos comerciales.

Los contrarios al prestamista de última instancia argumentan que si algunos bancos solventes enfrentan un shock que se traduce en problemas de liquidez, pueden recurrir al mercado interbancario que cumple la función de redistribuir la liquidez. De esta forma el mercado provee un seguro ante los shocks idiosincrásicos ${ }^{3}$. En el caso de perturbaciones agregadas que afectan a todos los 
bancos (no idiosincrásicas), se afirma que el Banco Central debería emitir a través de operaciones de mercado abierto, proveyendo liquidez al sistema como un todo, con el objeto de mantener la oferta monetaria a un nivel adecuado, pero debería abstenerse de apoyar directamente a los bancos con problemas. De acuerdo con esta posición, los bancos solventes pero ilíquidos podrían recurrir al mercado interbancario, mientras que los insolventes no conseguirían créditos y tenderían a desaparecer. Según este enfoque, la ayuda directa del Banco Central a los bancos comerciales se traduce en que estos asumen riesgos excesivos ante las expectativa de ser rescatados en situaciones adversas. Es decir, se genera un problema de riesgo moral ${ }^{4}$. El riesgo moral se refiere tanto a las acciones de los administradores de los bancos como a los depositantes, porque ambos pueden asumir riesgos excesivos ante la posibilidad de ayuda por parte del Banco Central.

Los argumentos a favor de que el Banco Central actúe como prestamista de última instancia toman como base la existencia de información imperfecta.

La información imperfecta puede producir una falla en el mercado de crédito interbancario ${ }^{5}$. Si no siempre es factible distinguir entre bancos solventes e insolventes, algunos bancos pueden obtener poco o ningún crédito en este mercado. Pero es posible contra argumentar que los bancos con problemas puedan ser de todas maneras ayudados por los otros bancos, si estos últimos quieren evitar que se produzca una situación de contagio, en que ellos mismos empiezan a perder depósitos. Sin embargo, hay dos limitaciones para esto. En primer lugar, es posible que algunos bancos se nieguen a participar en la ayuda, esperando que la situación la resuelvan otros bancos (problema de free riders). En segundo término, los montos involucrados pueden ser demasiado altos, por lo cual el problema no podría ser enfrentado sin ayuda del Banco Central ${ }^{6}$. Los partidarios del prestamista de última instancia encuentran así una justificación para que el Banco Central actúe para impedir el contagio y una crisis sistémica.

En el caso de una perturbación que afecta a todos los bancos (por ejemplo un shock macroeconómico), vimos que los contrarios a que el Banco Central actúe como prestamista de última instancia argumentan que este debe proveer liquidez a la economía en conjunto, a través de operaciones de mercado abierto ${ }^{7}$. Pero la justificación que se ha dado para que el Banco Central actúe como prestamista de última instancia, apoyando directamente a los bancos, es la mayor incertidumbre resultante de la quiebra de bancos importantes y de la inestabilidad del sistema bancario, con repercusiones negativas en el sector real. La incertidumbre generada haría a la demanda de dinero inestable e impredecible, lo que impediría que las

$4 \quad$ Véanse Bordo (1990) y Kaufman (1991).

5 Véanse Stiglitz y Weiss (1981),Claasen (1985), Freixas, Parigi y Rochet (1998), Goodhart y Huang (1999) y Freixas et alt. (2002).

6 En el caso de un país relativamente pequeño, los créditos de bancos extranjeros pueden ser una alternativa a la ayuda del Banco Central.

$7 \quad$ La posibilidad de que haya un shock macroeconómico es lo que Barandiarán, en su artículo en este mismo volumen, denomina riesgo exógeno. 
crisis se pudieran enfrentar a través de operaciones de mercado abierto que proveyeran liquidez al sistema como un todo manteniendo la oferta monetaria a un nivel adecuado $^{8}$.

Otro mecanismo para enfrentar los posibles problemas bancarios son los seguros de depósitos. Estos buscan evitar las corridas bancarias y así estabilizar al sistema bancario. Una posibilidad es que éstos fueran seguros privados. Pero es difícil que se puedan implementar estos seguros privados ${ }^{9}$. En primer lugar, existe el problema de determinar el riesgo que enfrentan los bancos y, por lo tanto, la prima que corresponde a ese nivel de riesgo. Para esto es necesario tener acceso a su contabilidad. Pero dicho requisito puede requerir tanto apoyo estatal que es posible que resulte más factible que los seguros sean directamente provistos por una agencia pública. Además, aun con acceso a la contabilidad puede persistir el problema de relacionar con exactitud las primas con el riesgo. Si este es el caso, el problema de riesgo moral resultante hace necesario aplicar controles a los bancos, $\mathrm{y}$ es posible que esto sólo pueda ser realizado en forma adecuada por el sector público. Por último, es muy importante considerar que si se produce una perturbación no idiosincrásica que afecta a todos los bancos nos encontraremos ante un riesgo agregado que no es fácil asegurar. En este caso sólo puede actuar el sector público, a través de su poder de cobrar impuestos, incluyendo el impuesto inflación.

Para que el seguro de depósitos elimine las corridas contra los bancos tendrían que estar asegurados todos los depósitos. Pero si este es el caso, se agrava el problema de riesgo moral. Así, tanto el seguro de depósitos como la función de prestamista de última instancia del Banco Central enfrentan el mismo dilema entre contagio y riesgo moral ${ }^{10}$.

Otro mecanismo que busca estabilizar el sistema bancario es la proposición de separar los bancos en dos organizaciones ${ }^{11}$. Una parte correspondería a los depósitos a la vista, contra las que se mantendría un encaje de $100 \%$. La otra tendría depósitos a plazo, con libertad respecto al tipo de activos. Pero es posible que se desarrollen formas en que las instituciones no sometidas a controles comiencen a ofrecer servicios de transacción similares a las que proveen los depósitos a la vista ${ }^{12}$. Además, y esta es la limitación más importante, los problemas de los bancos no surgen sólo del retiro de depósitos a la vista, sino que también de la no renovación de depósitos a plazo. reciente sobre el riesgo de crisis en el sector bancario, véase De Brand y Hartmann (2002).

9 Véanse Diamond y Dybvig (1983), Claassen (1985) y Goodhart (1993).

10 Existen otras alternativas a los préstamos de última instancia. Una alternativa es la modificación temporal de algunas restricciones, como, por ejemplo, los requisitos de encaje. Para esta y otras alternativas posibles, véase el artículo de Barandiarán en este mismo volumen.

11 Véase Freixas y Rochet (1997).

12 Véase Goodhart (1993). 
La controversia entre las dos posiciones respecto al prestamista de última instancia continúa. Una posición pone el énfasis en el riesgo moral que genera el Banco Central, y la otra la necesidad de evitar el contagio entre bancos y los efectos negativos generados por las quiebras de bancos importantes ante un shock macroeconómico. Pero en la práctica los bancos centrales, de hecho, actúan como prestamistas de última instancia, algunos desde hace más de un siglo. En las siguientes dos secciones analizaremos la visión clásica sobre la forma en que los bancos centrales deberían llevar a cabo su labor de prestamistas de última instancia, y también la forma cómo efectivamente operan en general.

\section{La Discusión Clásica: Thorton y Bagehot ${ }^{13}$}

El término "prestamista de última instancia” se originó en Baring (1797), quien se refirió al Banco de Inglaterra como la "última instancia" a la cual los bancos podrían recurrir para obtener liquidez en una crisis.

Sin embargo, el primero en tratar el tema del prestamista de última instancia en detalle y con cierto rigor fue Thorton (1802). Cuando Thorton publicó su libro en 1802, llevaba ya años analizando los problemas monetarios y bancarios, en especial las quiebras de bancos y los acontecimientos monetarios que llevaron a la suspensión de la convertibilidad en 1797. La convertibilidad de los billetes del Banco de Inglaterra en oro sólo se restableció en 1821.

Thorton argumentó que el Banco de Inglaterra debía velar por la estabilidad del sistema bancario, proveyendo liquidez a los bancos en problemas para evitar un pánico generalizado. Esta visión del Banco de Inglaterra, como un Banco Central con responsabilidad respecto al sistema bancario en conjunto, no era compartida por los directores del banco, quienes ponían el énfasis en el negocio bancario propiamente tal y en la obtención de utilidades.

La proposición de Thorton fue que en el caso de una crisis de confianza que se tradujera en retiro de depósitos, el Banco de Inglaterra debía prestar en forma liberal a los bancos solventes, para restablecer y mantener la confianza.

Junto con argumentar a favor de la función de prestamista de última instancia, Thorton relacionó esto con la política monetaria. Señaló que debería seguirse una política cuidadosa en relación con la emisión, de manera tal de no perder excesivas reservas (bajo tipo de cambio fijo) o no causar inflación (bajo tipo de cambio flexible $)^{14}$. Pero la función de apoyar a los bancos ante una crisis de confianza parecería incompatible con una política de crecimiento moderado de la emisión. Sin embargo, Thorton afirmó que no existe conflicto entre estos dos objetivos, ya que si el Banco Central reacciona en forma oportuna se puede evitar un pánico generalizado, e incluso la mera expectativa de intervención del instituto 
emisor puede frenar el pánico, sin que sea necesario un aumento muy significativo de la base monetaria. Esto se traduciría en que la oferta monetaria no se desviaría mucho de la meta de largo plazo.

Thorton también fue el primero en explicitar claramente el problema de riesgo moral y el dilema entre este y el peligro de contagio. En relación con esto, argumentó que no debería apoyarse a los bancos cuyos problemas surgieron de una conducta poco prudente, aunque indicó que es difícil alcanzar un justo medio entre el riesgo moral y la posibilidad de una crisis sistémica que haga necesario apoyar aun a bancos poco prudentes.

El otro gran aporte a la discusión clásica sobre este tema fue el libro de Bagehot (1873), quien aceptó y extendió las ideas de Thorton. Igual que Thorton, Bagehot era partidario de que el Banco de Inglaterra actuara como prestamista de última instancia para impedir las crisis sistémicas en el sector bancario. Según Bagehot, el prestamista de última instancia debía operar de acuerdo a los principios que se indican a continuación.

En primer lugar, debía prestar en forma liberal pero a una tasa de interés alta, por encima de la tasa de interés de mercado.

En segundo término, sólo debía prestar a los bancos con problemas de liquidez pero solventes. Estos préstamos debían hacerse contra activos de buena calidad. Por supuesto que en una situación de crisis estos activos bajarían de precio, pero Bagehot argumentó que el Banco Central debería considerarlos a su valor "normal", es decir, al valor que tendrían una vez superada la crisis. En ningún caso deberían hacerse préstamos contra activos de mala calidad, que resultaran eventualmente en pérdidas para el Banco de Inglaterra. Bagehot argumentó, con bastante optimismo, que los activos de mala calidad constituían una pequeña proporción del total. Según esto, predominarían los problemas de liquidez sobre los de solvencia.

En tercer lugar, el Banco de Inglaterra debía publicitar su disposición a actuar de la manera especificada en los dos puntos anteriores. El conocimiento anticipado de esto impediría los pánicos generalizados.

\section{El Prestamista de Ultima Instancia en la Práctica ${ }^{15}$}

En el análisis clásico el prestamista de última instancia sólo debe apoyar a los bancos solventes pero con problemas de liquidez. Esto presupone que es posible distinguir claramente los bancos solventes de los insolventes, lo que en la práctica muchas veces no se cumple.

Un banco claramente solvente que experimenta problemas de liquidez puede obtener créditos en el mercado interbancario. Los problemas de liquidez que no 
se resuelven a través de mecanismos de mercado muchas veces van acompañados de problemas de solvencia.

En todo caso, porque no siempre se puede distinguir entre bancos solventes e insolventes, surge la función de prestamista de última instancia, que apoya al que nadie más le quiere prestar. Pero si este es el caso, no se puede seguir la regla de prestarle sólo a los solventes pero ilíquidos. Sólo después de los hechos se va a saber plenamente cuál era la situación exacta de un banco al que se apoya, ante el temor de que su quiebra genere contagio y una crisis sistémica. Además, lo más probable es que el Banco Central enfrente pérdidas, ya que al ser el prestamista de última instancia es muy poco probable que preste contra activos de buena calidad, como recomendaba Bagehot.

Según el enfoque clásico, el Banco Central debía prestar en forma liberal pero a una tasa de interés bastante alta. Respecto a la tasa de interés elevada, en los casos de apoyo a bancos con alta probabilidad de generar una crisis sistémica esto, en general, no se aplica, ya que la preocupación principal es evitar el contagio $^{16}$.

En algunos casos el Banco Central enfrentará restricciones para "prestar en forma liberal". En primer lugar, si existe convertibilidad en un bien (como en el patrón oro) o en una moneda extranjera, la función de prestamista de última instancia está bastante limitada. Si existe tipo de cambio flexible el Banco Central puede movilizar recursos en forma importante. Pero si se tienen objetivos de control de la inflación, aun bajo tipo de cambio flexible existirán limitaciones a los préstamos que puede hacer el instituto emisor.

El alto volumen de los montos involucrados en las crisis bancarias que se han generado a partir de la década de 1930 se ha traducido en que los bancos centrales no han podido apoyar a los bancos sólo con sus propios recursos. En algunos casos los bancos centrales han coordinado un apoyo concertado, en el que han participado algunos bancos comerciales, aportando fondos junto al Banco Central para apoyar a los bancos en problemas. Esto era más fácil cuando existían "clubes" de bancos cartelizados, y era más factible para el Banco Central obtener la cooperación de algunos bancos. Pero este mecanismo se ha debilitado en los últimos años, como consecuencia de la desregulación y la mayor competencia, especialmente a nivel internacional.

Los montos importantes involucrados en algunas crisis bancarias también han significado que, en muchas oportunidades, los bancos centrales han sido el agente técnico en las operaciones de rescate, pero con recursos aportados por el gobierno, el que puede actuar como prestamista de última instancia gracias a su capacidad de cobrar impuestos. La participación del gobierno ha aumentado a partir de la década de 1980, dado el tamaño de las crisis bancarias, muchas de ellas generadas por shocks macroeconómicos ${ }^{17}$.

16 Véase Giannini (1999)

17 Véase Goodhart y Schoemaker (1993) para un análisis de 104 casos de bancos en problemas en 24 países, en el período 1970-1992. En la mayoría de los casos los bancos no fueron apoyados por el banco central solo, sino que con la ayuda de otros bancos o de otras instituciones públicas. 
En la práctica, en los diferentes países normalmente se ha apoyado a los bancos en problemas después de la década de 1930. Sólo como excepción se ha dejado quebrar bancos, en general pequeños, ya que los grandes casi siempre son apoyados. Esto ha generado un problema de riesgo moral.

Para enfrentar el problema de riesgo moral, se ha puesto el énfasis en la supervisión bancaria y se han impuesto controles directos a la toma de riesgos, tales como requerimientos de capital y de estructura de activos, revisiones, etc. La supervisión provee también información que puede ayudar a las autoridades a discriminar entre bancos en caso de crisis. También se ha buscado incentivar la supervisión privada de los bancos. Para esto se ha excluido del seguro de depósitos a los inversionistas grandes, los que tienen mayores incentivos y medios para analizar la situación de los bancos.

Hemos visto que el tercer principio de Bagehot era que el Banco Central debía publicitar su disposición a ayudar a los bancos. Sin embargo, el problema de riesgo moral ha llevado a los bancos centrales a aplicar una política que se ha denominado "ambigüedad constructiva". Esta consiste en que no se explicita claramente si se va a apoyar o no a los bancos con problemas, guardándose el instituto emisor la facultad de actuar caso a caso en forma discrecional, con la esperanza de que esto disminuya el riesgo moral.

Como hemos visto que en la mayoría de los casos los bancos centrales han apoyado a los bancos comerciales en problemas, un aspecto importante de la "ambigüedad constructiva" es que no se sabe cuáles serán las condiciones que acompañarán a la posible ayuda ${ }^{18}$. Aun cuando se apoye a un banco para evitar su quiebra, la ayuda puede ir acompañada de sanciones que pueden ser aplicadas en diferentes grados, y que pueden traducirse en remoción de administradores y pérdidas para los accionistas y depositantes. Esto, por supuesto, está relacionado con la regulación y supervisión bancaria y con la información y herramientas que éstas proveen a las autoridades.

Podemos concluir que, en la práctica, los bancos centrales actúan como prestamista de última instancia en un contexto más complejo que el visualizado en el enfoque clásico, y en el que está siempre presente el dilema entre contagio y riesgo moral. Esto los ha llevado a poner el énfasis en la supervisión bancaria, efectuada directamente por el Banco Central o a través de otra institución, y a aplicar una política de "ambigüedad constructiva", en lugar de actuar a través de reglas claras y transparentes. del banco central a los bancos comerciales, contingente a una perturbación agregada, aumenta el valor de los bancos como empresas que continúan existiendo. Los autores argumentan que esto puede generar incentivos para actuar en forma prudente, más que compensando el problema de riesgo moral que introduce el seguro provisto por el banco central. 


\section{Crisis Financieras y el Prestamista de Ultima Instancia INTERNACIONAL}

Las crisis financieras internacionales de los años recientes han motivado una serie de proposiciones para reformar el sistema financiero internacional y crear una "nueva arquitectura financiera internacional" 19 . Esto, con el objetivo de disminuir el número e intensidad de las crisis financieras (prevención de crisis) y desarrollar respuestas adecuadas cuando éstas se presentan (manejo de crisis).

Las proposiciones que se han hecho para dar más estabilidad al sistema financiero internacional se refieren a los sectores privado y público de los países receptores y de los originarios de los flujos internacionales de capital, como asimismo a las instituciones financieras internacionales (Fondo Monetario Internacional, Banco Mundial, Banco de Pagos Internacionales, etc. $)^{20}$

Respecto de la función del Fondo Monetario Internacional en la prevención y manejo de las crisis financieras, ésta incluye tres aspectos: la supervisión, la difusión de información y el financiamiento.

En relación con el financiamiento ha surgido la discusión sobre si el Fondo Monetario Internacional debe actuar como prestamista de última instancia internacional. Aquí la cuestión fundamental es el dilema entre crisis sistémicas y riesgo moral, igual como ocurre a nivel nacional. El Fondo Monetario Internacional ha sido criticado con el argumento de que, al rescatar a los gobiernos y a los inversionistas privados, ha creado problemas de riesgo moral, incentivando el otorgamiento temerario de créditos, y fomentando así nuevas crisis financieras. El dilema es entonces entre "rescates de rutina", que generan riesgo moral, y la posibilidad de importantes crisis sistémicas internacionales. El camino intermedio es realizar en forma ordenada la reestructuración de deudas con problemas, con un papel activo del Fondo Monetario Internacional. Esta reestructuración ordenada puede ser complementada con el apoyo financiero de esta institución.

Aunque la función de prestamista de última instancia a nivel internacional nos enfrenta al familiar dilema entre riesgo moral y contagio, además esta función se realiza en un contexto indudablemente más complejo que el que existe a nivel nacional, lo que introduce importantes problemas adicionales ${ }^{21}$.

\footnotetext{
19 Esta sección se basa en parte en Ossa (2002).
}

20 Véanse Council of Foreign Relations (1999), Eichengreen (1999), Fischer (1999) y (2000), Godhart y Huang (2000), De Gregorio (2002) y Goodhart e Illing (2002), volumen, el cual se centra en el tema al que hemos hecho referencia en esta última sección. 


\section{REFERENCIAS}

Bagehot, W. (1873), Lombard Street. A Description of the Money Market, Keegan, Paul \& Co., London.

Baring, F. (1797), Observations on the Establishment of the Bank of England and on the Paper Circulation for the Country. To Which is Added Further Observations. Reimpreso en 1967, Kelley, New York.

Bordo, M. (1990), The Lender of Last Resort: Some Historical Insights, NBER Working Paper 3011.

Broda, C. y E. Levy-Yeyati (2002), "Dollarization and the Lender of Last Resort", en E. Levy-Yeyati y F. Sturzenegger (eds.), Dollarization, MIT Press, Cambridge, Mass.

Claassen, E.M. (1985), “The Lender of Last Resort Function in the Context of National and International Crisis", Weltwirschaftliches Archiv 121(2): 217-237.

Cordella, T. y E. Levy-Yeyati (1999), Bank Bailouts: Moral Hazard vs Value effect, IMF Working Paper, 99/106.

Council of Foreign Relations (1999), Task Force Report on Safeguarding Prosperity in a Global Financial System. The Future of International Financial Architecture, Institute for International Economics, Washington, D.C.

De Brand, O. y P. Hartmann (2002), "Systemic Risk in Banking: A Survey”, en C.A.E. Goodhart y G. Illing (eds.), Financial Crises, Contagion, and the Lender of Last Resort, Oxford University Press, Oxford and New York.

De Gregorio, J. (2002), Macroeconomic Management in Emerging Economies and the International Financial Architecture, Documento de Trabajo $N^{\circ} 163$, Banco Central de Chile.

Diamond, D.W. y P.H. Dybvig (1983), "Bank Runs, Deposit Insurance and Liquidity", Journal of Political Economy, 91(3): 401-419.

Dowd, K. (1994), “Competitive Banking, Banker's Clubs, and Banking Regulation”, Journal of Money, Credit and Banking, 26(2): 289-308.

Eichengreen, B. (1999), Toward a New International Financial Architecture, Institute for International Economics, Washington, D.C.

Fischer, S. (1999), "Reforming the International Financial System", Economic Journal, 109: $557-576$.

Fischer, S. (2000), On the Need for an International Lender of Last Resort, Princeton Essays in International Economics $\mathrm{N}^{\circ} 220$.

Freixas, X. et al. (2002), "Lender of Last Resort: A Review of the Literature", en C.A.E. Goodhart y G. Illing (eds.), Financial Crises, Contagion, and the Lender of Last Resort, Oxford University Press, Oxford and London.

Freixas, X. y J.C. Rochet (1997), Microeconomics of Banking, MIT Press, Cambridge, Mass.

Freixas, X., B. Parigi y J.C. Rochet (1998), The Lender of Last Resort: A Theoretical Foundation, mimeo.

Fry, M., C.A.E. Goodhart y A. Almeyda (1996), Central Banking in Developing Countries: Objectives, Activities and Independence, Routledge, London and New York.

Giannini, C. (1999), Enemy of None but Common Friend of All? An International Perspective on the Lender of Last Resort, Princeton Essays on International Finance $\mathrm{N}^{\circ} 214$.

Goodhart, C.A.E. (1988), The Evolution of Central Banks, MIT Press, Cambridge, Mass. 
Goodhart, C.A.E. (1993), "Bank Insolvency and Deposit Insurance: A Proposal”, en P. Arestis (ed.), Money and Banking: Issues for the Twenty First Century, MacMillan, London.

Goodhart, C.A.E. (1995), The Central Bank and the Financial System, MIT Press, Cambridge, Mass.

Goodhart, C.A.E. (2002), "Myths about the Lender of Last Resort", en C.A.E. Goodhart y G. Illing (eds.), Financial Crises, Contagion, and the Lender of Last Resort, Oxford University Press, Oxford and New York.

Goodhart, C.A.E. y D. Schoemaker (1993), "Institutional Separation Between Supervisory and Monetary Agencies", en F. Bruni (ed.). Prudential Regulation, Supervision and Monetary Policy, Universita Bocloni, Milano.

Goodhart, C.A.E. y H. Huang (2000), A Model of the Lender of Last Resort, IMF Working Paper 99/39.

Goodhart, C.A.E. y G. Illing (eds.) (2002), Financial Crises, Contagion and the Lender of Last Resort, Oxford University Press, Oxford and New York.

Gorton, G. y D.J. Mullineaux (1987), “The Joint Production of Confidence: Endogenous Regulation and Nineteenth Century Commercial Bank Clearing Houses", Journal of Money, Credit and Banking, 19(4): 457-468.

Hernández, L. y K. Schmidt-Hebbel (eds.) (2002), Banking, Financial Integration, and International Crises, Banco Central de Chile, Santiago.

Humphrey, T.M. y R.E. Keleher (1984), "The Lender of Last Resort. A Historical Perspective”, Cato Journal 4(1): 275-318.

Kaufman, G.G. (1991), "Lender of Last Resort: A Contemporary Perspective", Journal of Financial Services Research, 5(2): 95-110.

Ossa, F. (2002), Sistemas Monetarios Nacionales e Internacionales, Ediciones Universidad Católica de Chile, Santiago.

Schuler, K. (1996), Should Developing Countries Have Central Banks? Currency Quality and Monetary Systems in 155 countries. Research Monograph $N^{\circ}$ 52, Institute of Economic Affairs, London.

Stiglitz, J.E. y A. Weiss (1981), “Credit Rationing in Markets with Imperfect Information”, American Economic Review, 71: 393-410.

Thorton, H. (1802), An Inquiry into the Nature and Effects of the Paper Credit of Great Britain, edited with an introduction by F.A. von Hayek (1939), George Allen and Unwin, 1939. 